\title{
Morphogenesis of Mycoplasma and Bacterial L-form Colonies
}

\author{
By S. RAZIN AND OFRA OLIVER \\ Department of Clinical Microbiology, Hebrew University-Hadassah Medical. \\ School, Jerusalem, Israel
}

(Received 8 August 1960)

\begin{abstract}
SUMMARY
The development and mechanism of formation of Mycoplasma and bacterial L-form colonies were studied. The micro-organisms penetrate into the agar within a few hours after inoculation. It is suggested that penetration is caused by capillary forces which draw the minute plastic organisms into the dried agar gel, together with the water surrounding them. Penetration does not take place when the agar surface is very moist. The organisms appear to develop in the interstices of the fibrillar network of the agar gel and form a firm and elastic ball-like colony growing in all directions. When reaching the agar surface the growth spreads into the thin free water film which covers the agar, forming the peripheral zone typical of mature colonies. Factors which decrease the thickness of the free water film, like drying the medium or increasing the agar concentration, cause a decrease of the peripheral zone dimensions. Factors which retard growth, such as high concentrations of inorganic salts or hydrogen ions, inhibit the formation of the peripheral zone by limiting the initial ball-like growth inside the agar, rendering it incapable of reaching the agar surface. Typical colonies composed of both central and peripheral zones appear also on other fibrillar media such as the cellulose pellicle of Acetobacter xylinum and gelatin.
\end{abstract}

\section{INTRODUCTION}

Mycoplasma organisms (PPLO) and the L-forms of bacteria share a typical colony morphology, the 'fried-egg' colony. This colony is made up of a central zone, embedded in the agar and a peripheral zone on the agar surface (Klieneberger-Nobel, 1960; Dienes, 1960). The morphology of the colony, unique for these organisms, serves as one of the most important criteria in distinguishing them from other bacteria. Although much work has been done on the morphology of Mycoplasma and L-forms, the information concerning the mode of formation of the colonies is scanty (Klieneberger, 1934; Dienes, 1945). Our aim was, therefore, to study the development of these peculiar colonies and the mechanisms involved in their formation.

Two features shared by the Mycoplasma and L-forms seem to be responsible for the formation of the 'fried-egg' colonies: the very small dimensions $(100-300 \mathrm{~m} \mu)$ of the minimal reproductive units (Klieneberger-Nobel, 1956) and their plasticity due to the absence of a rigid cell wall (Klieneberger-Nobel, 1960). 


\section{METHODS}

Organisms. Mycoplasma laidlawii strain A (PG8), M. laidlawii strain B (PG9), M. bovigenitalium (PG11), M. gallisepticum (PG 31), M. mycoides var. mycoides (PG 1), M. neurolyticum (PG 28) and the stable L-form of Proteus were kindly given by Dr D. G. ff. Edward (The Wellcome Research Laboratories, Beckenham, Kent). $M$. mycoides var. capri and the stable L-form of Streptobacillus moniliformis were the gift of Dr E. Klieneberger-Nobel (The Lister Institute for Preventive Medicine, London). M. hominis was isolated in our laboratory from a case of vaginitis.

Media. The medium used for stock cultures and most experiments was a modified Edward medium (Butler \& Knight, 1960 a) where pooled inactivated human serum replaced horse serum. Beef heart for infusion, peptone and yeast extract used for compounding Edward medium were products of Difco Laboratories Inc. (Detroit 1, Michigan, U.S.A.). Following the suggestion of Klieneberger-Nobel (1956) the medium was enriched by addition of a nutrient broth culture filtrate of Staphylococcus aureus in a final concentration of $12.5 \%(\mathrm{v} / \mathrm{v})$. Edward medium was solidified with $1.25 \%$ $(\mathrm{w} / \mathrm{v})$ Bacto-agar (Difco, certified) except for some experiments where $12 \%(\mathrm{w} / \mathrm{v})$ Bacto-gelatin (Difco) was used.

Coagulated serum medium (Löffler's medium) was prepared by mixing three parts of bovine serum with one part of Difco nutrient broth containing $1 \%(w / v)$ glucose. The medium was poured into Petri dishes and sterilized by inspissation at $85^{\circ}$ for $2 \mathrm{hr}$.

Cellulose medium. Acetobacter xylinum was grown on the medium of Hestrin \& Schramm (1954) for 2 days at $30^{\circ}$. The thick pellicle formed was removed, washed thoroughly with sterile distilled water and immersed in sterile distilled water at $6^{\circ}$ for 3 days. The water was changed daily. The white cellulose pellicle was then soaked in liquid Edward medium, transferred to a filter paper disk to remove excess liquid and finally placed on the surface of solid Edward medium.

Conditions of growth. Test tubes $\left(6 \times \frac{5}{8}\right.$ in.) containing $5 \mathrm{ml}$. of liquid Edward medium were inoculated with $0.1 \mathrm{ml}$. of the stock cultures and incubated statically in air at $37^{\circ}$. Optimal growth of all strains occurred usually after $48 \mathrm{hr}$. incubation. Decimal dilutions of these broth cultures were made in a solution of $1 \%(\mathrm{v} / \mathrm{v})$ Edward medium in 0.01 M-K $\mathrm{K}_{2} \mathrm{HPO}_{4}$ buffer ( $\mathrm{pH} \mathrm{8.0;}$ Butler \& Knight, 1960b). Standard drops ( $0.02 \mathrm{ml}$. each) of these dilutions were placed on solid medium plates which had previously been dried by leaving them open in the $37^{\circ}$ incubator for $1-1.5 \mathrm{hr}$. The dilutions used for inoculation were chosen so as to give no more than 500 colonies per drop area. The plates were placed in closed tins containing moist cotton wool and incubated at $37^{\circ}$ for 3 days.

Measurement of colony and central zone diameters. The growth which appeared on plates was inspected by means of an ordinary light microscope, using slightly oblique transmitted light. Measurement of colony and central zone diameters was carried out with a $\times 10$ objective and $a \times 5$ ocular fitted with a micrometer disk. The drop area was divided by a line through its centre cut by means of a razor blade, and 50 colonies were measured along this line, assuring the representation of colonies growing in the periphery and centre of the drop area.

Vertical sections of colonies. Large colonies were selected for this purpose. The section was cut through the centre of the colony by means of a razor blade. For good 
results the thickness of the section had to be not greater than $0.2-0.3 \mathrm{~mm}$. The sections were transferred to slides and examined by the light microscope.

Technique for demonstrating penetration of micro-organisms into the agar. Standard drops of diluted suspensions of the organisms tested were placed on the surface of dried Edward medium plates. Immediately after the drops had dried and at various time intervals later, small agar squares (larger than a drop area) were cut from the Edward medium and placed on the drop areas, care being taken not to form air bubbles. The agar slices were removed, turned over and placed on slides. The agar plates and slices were then incubated at $37^{\circ}$ for 3 days in a moist atmosphere. The number of colonies appearing in the drop areas on the plates and on the corresponding agar slices was determined by using the light microscope (magnification $\times 25)$.

\section{RESULTS}

\section{The development of Mycoplasma and bacterial L-form colonies}

Edward medium plates were inoculated with suspensions of Mycoplasma laidlawii strain $A$ and the L-form of Streptobacillus moniliformis. Visible colonies appeared after 24-hr. incubation at $37^{\circ}$ (Pl. 1, figs. 1, 4). At this phase of growth almost all colonies in both organisms appeared to be small spheres embedded in agar. This was proved by the circular shape of vertical sections through the colonies and by the inability to remove the colonies on scraping the agar surface with a razor blade.

Further incubation for another $24 \mathrm{hr}$. caused enlargement of Mycoplasma laidlazvii strain A colonies, some of which had already developed the peripheral zone (Pl. 1, fig. 2). The L-form grew faster than the Mycoplasma and almost all colonies developed into the 'fried egg' type after $48 \mathrm{hr}$. incubation (Pl. 1, fig. 5). The peripheral zone of the colonies, located on the agar surface, was easily removed by scraping. Mature colonies of both organisms may be seen in Pl. 1, figs. 3, 6. These colonies had big peripheral zones.

The development of colonies was also followed quantitatively by measuring the diameter of the colonies and their central zones (Fig. 1). The central zone was the first to develop, followed by the formation of the peripheral zone later on. The central zone grew only a little after $48-\mathrm{hr}$. incubation, whereas the peripheral zone continued to grow, until it occupied the greater part of the colony after incubation for $96 \mathrm{hr}$.

\section{Vertical sections of mature colonies}

All Mycoplasma and L-form strains used in this investigation were examined. The colony sections showed the same basic structure in all organisms: a hemispherical central zone which is embedded in the agar and a thin peripheral zone which spreads over the agar surface (Pl. 2, figs. 7-10). The central zone was a firm and elastic structure in comparison with the very soft peripheral zone.

\section{Penetration of Mycoplasma and L-forms into the agar and formation of the central zone}

In order to understand the mechanism of formation of Mycoplasma and L-form colonies we thought it worth while to investigate why the growth of these organisms begins in the agar. Experiments to this purpose showed that the organisms penetrated into the agar medium before multiplication. As may be seen from 
Table 1, immediately after the drops had dried about $50 \%$ of the Mycoplasma organisms could be transferred to the agar slices. Further incubation gradually decreased the number of organisms that could be transferred and at 6-8 hr. after inoculation almost all organisms remained on the agar plate.

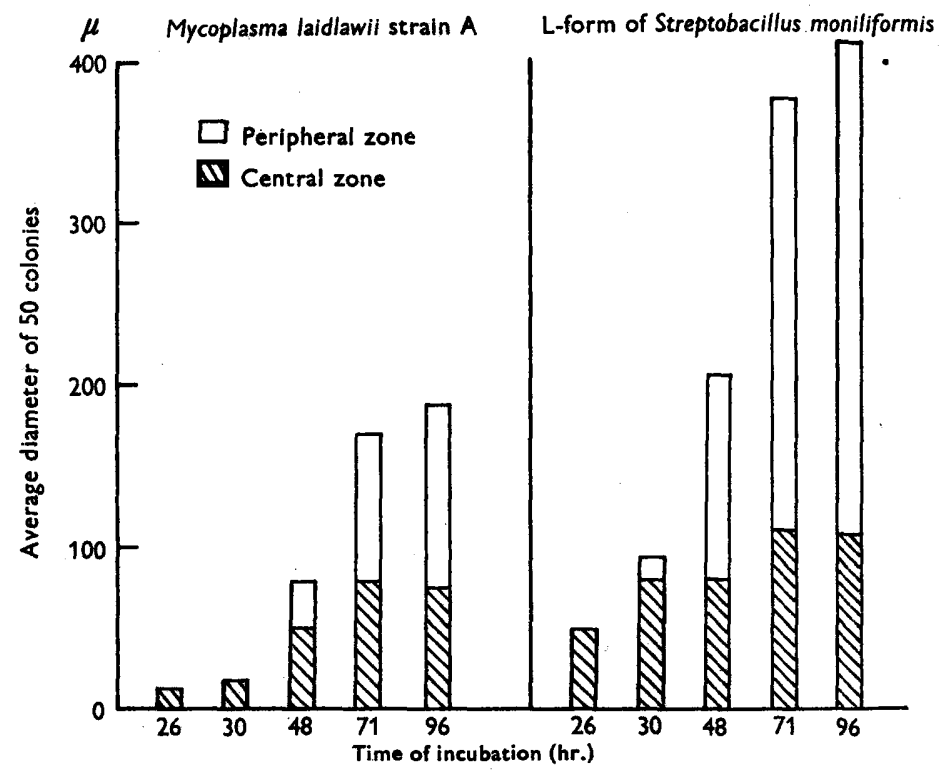

Fig. 1. The development of colonies of Mycoplasma laidlawii strain A and the L-form of Streptobacillus moniliformis on solid Edward medium.

The sum of the number of colonies which developed on the drop area on the plate and the corresponding agar slice remained constant within the first $8 \mathrm{hr}$. of experiment, indicating that no mortality or multiplication took place during this period. After 24-hr. incubation the number of colonies which appeared on the agar slices increased and became equal to the number of colonies on the corresponding drop areas. However, in most experiments the growth was confluent. This was due to the smear of the surface peripheral zones which began to appear at this time ( $\mathrm{Pl} .1$, figs. 2, 4).

The four Mycoplasma strains tested behaved similarly; almost all organisms penetrated into the agar within 6-8 hr. after inoculation. The L-forms of Proteus sp. and Streptobacillus moniliformis differed in this respect since the number of organisms which could be transferred to the agar slice did not change significantly during the first $8 \mathrm{hr}$. of the experiment.

For comparison the same experiment was repeated with Staphylococcus aureus and Streptococcus pyogenes. More than $50 \%$ of the organisms inoculated could be transferred to the agar slices after 3-hr. incubation, indicating that these organisms did not penetrate into the agar.

Mycoplasma penetration into the agar did not occur when the surface of the agar was moist. Moist Edward agar plates were prepared by pouring different amounts of sterile distilled water on the agar surface. With Mycoplasma laidlawii strain A as 


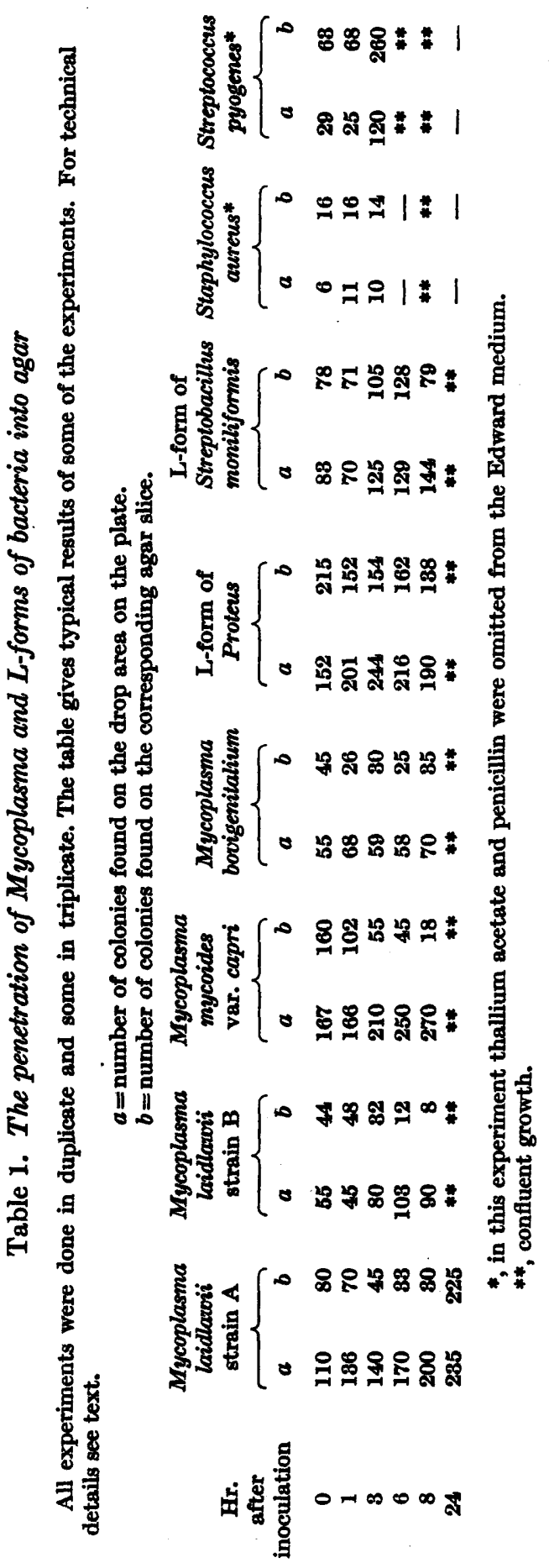


inoculum, growth was inspected after incubation at $37^{\circ}$ for 3 days in a moist atmosphere. The colonies which appeared on the surface of the moist plates were large, flat, and had either minute central zones or none at all (Pl. 2, figs. 11, 12).

Suspensions of Mycoplasma or L-forms were mixed with melted Edward agar at $45^{\circ}$ and poured into plates. Most colonies developed deep inside the agar, forming ball-like structures, in contrast to the lens-like deep agar colonies of Eubacteria such as Staphylococcus aureus (Pl. 3, figs. 13-15).

\section{Factors influencing the form and dimensions of the peripheral zone}

It was assumed that the peripheral zone of Mycoplasma and L-form colonies develop in the thin free water film which is present on the agar surface (Knaysi, 1951). This view was supported by the demonstration of the spreading of peripheral zones along cotton-wool fibres placed on the agar (Pl. 3, figs. 16, 17).

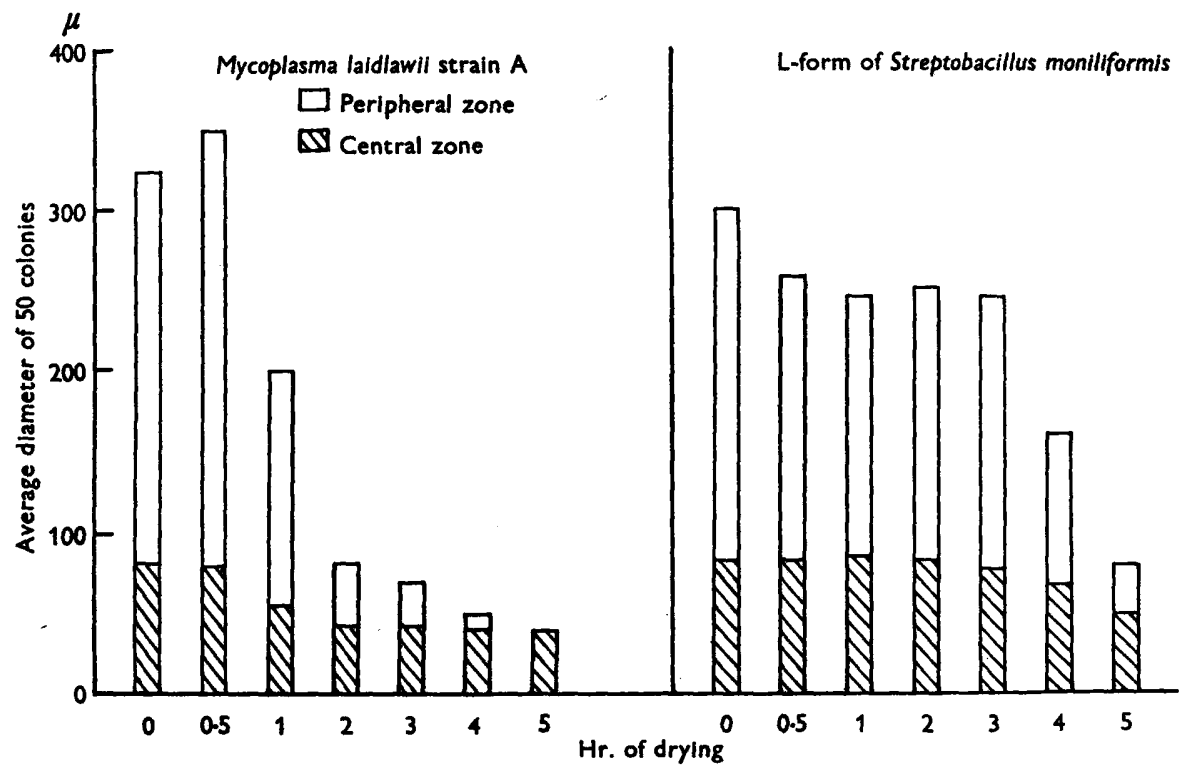

Fig. 2. The effect of pre-drying the Edward medium on the morphology of colonies of Mycoplasma laidlawii strain A and the L-form of Streptobacillus moniliformis.

The need of a humid atmosphere for optimal growth of Mycoplasma and L-forms has been stressed by many authors (Edward, 1954; Klieneberger-Nobel, 1954). Drying of the plates and subsequent incubation in a dry atmosphere decreases the free water film on the agar surface, possibly affecting the formation of the peripheral zones. To test this assumption, solid Edward medium in Petri dishes was dried for different periods of time by opening the plates in a $37^{\circ}$ incubator. After the inoculation with suspensions of Mycoplasma laidlawii strain A and the L-form of Streptobacillus moniliformis the plates were placed in tins without moist cotton wool and incubated at $37^{\circ}$ for 3 days. The dimensions of the peripheral zones were found to be markedly decreased in the case of the dried plates (Fig. 2). Drying for $5 \mathrm{hr}$. caused complete disappearance of the peripheral zones in all colonies of $M$. laidlawii strain $\mathbf{A}$ 
and in almost all colonies of the L-form of $S$. moniliformis. The rate of reduction of the central zone diameter due to drying of the medium was much smaller than that of the peripheral zone diameter.

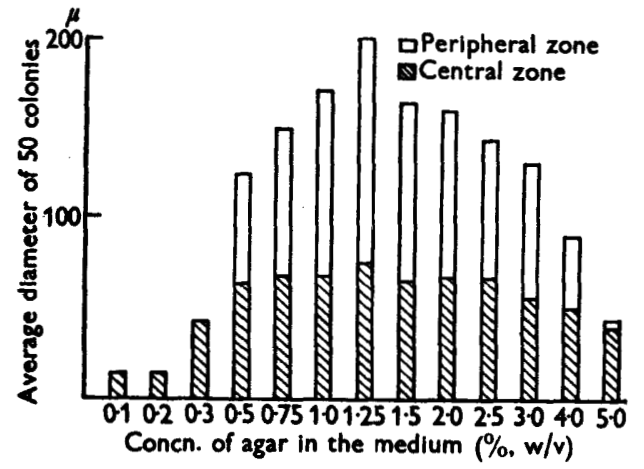

Fig. 3

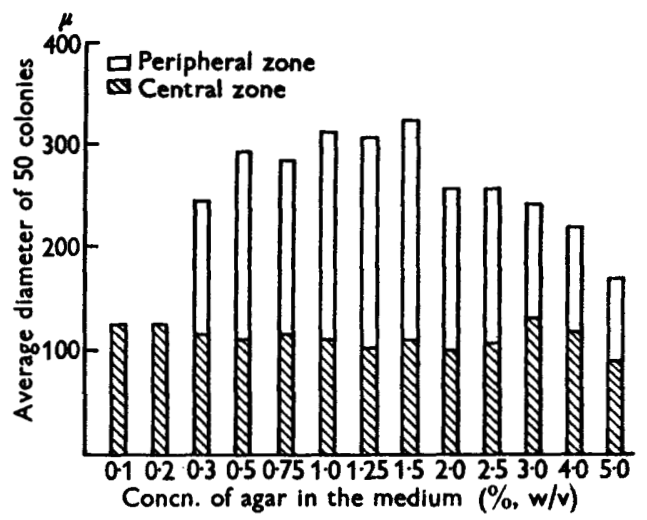

Fig. 4

Fig. 3. The effect of agar concentration in Edward medium on the morphology of Mycoplasma laidlawii strain A colonies.

Fig. 4. The effect of agar concentration in Edward medium on the morphology of colonies of the L-form of Streptobacillus moniliformis.

\section{The effect of agar concentration on the morphology of the colonies}

The effect of changes in the agar concentration in Edward medium on the morphology of colonies of Mycoplasma laidlawii strain A and the L-form of Streptobacillus moniliformis is illustrated in Figs. 8 and 4 . With low agar concentrations $(0 \cdot 1-0 \cdot 3 \%, w / v)$, the medium was semisolid and $M$. laidlawii strain A grew in small irregular ball-like colonies at various depths of the medium. A hazy surface growth was also present. Typical colonies, consisting of both central and peripheral zones, appeared at a concentration of $0.5 \%(w / v)$. Increasing the concentration of the agar to $1.25 \%(w / v)$ increased the diameter of the colonies, mainly that of the peripheral zones. Further increase in agar concentration caused a gradual decrease of the colony diameter, affecting primarily the peripheral zone. At a concentration of $\mathbf{5} \%(w / v)$ agar, almost all colonies were without peripheral zones. Similar results were obtained with the L-form of 5 . moniliformis (Fig. 4). The diameter of the central zone of the L-form colonies did not change significantly by changing the agar concentration of the medium. The optimal agar concentration for the development of the peripheral zone was about $1.5 \%(w / v)$; higher concentrations inhibited its formation. A hazy surface growth was observed with low agar concentrations $(0 \cdot 1-0 \cdot 2 \%, w / v)$.

\section{Morphology of Mycoplasma colonies on solid media other than agar}

Mycoplasma laidlawii strains A and B and $M$. mycoides var. capri were grown on Edward medium solidified with $12 \%(\mathrm{w} / \mathrm{v})$ gelatin instead of agar. The plates were incubated for 3 days at $25^{\circ}$. Growth appeared mostly in the shape of balllike colonies at various depths of the medium. Some colonies near the surface also 
developed peripheral zones. Liquefaction of the gelatin by the organisms after further incubation masked this typical colony morphology.

Mycoplasma laidlawii strain A gave rise to colonies composed of central and peripheral zones when grown on the cellulose pellicle of Acetobacter xylinum. Many colonies, however, had no peripheral zones since they had developed deep inside the pellicle, resembling the deep agar colonies of Mycoplasma (Pl. 3, fig. 13).

Mycoplasma laidlawii strain A gave flat surface colonies without central zones when grown on coagulated serum medium. The same type of surface colonies, not penetrating into the medium, appeared when this organism was grown on the surface of a cellophan dialysis membrane placed on solid Edward medium.

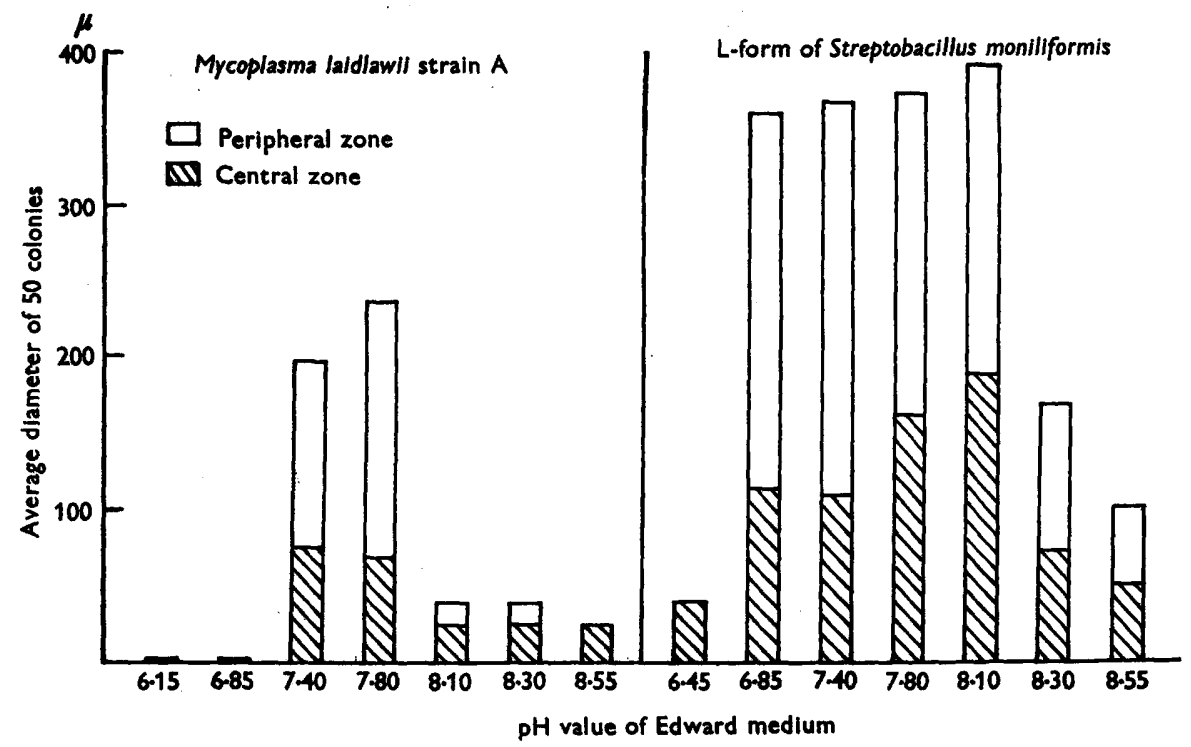

Fig. 5. The effect of $\mathrm{pH}$ value on the growth of Mycoplasma laidlawii strain A and the L-form of Streptobacillus moniliformis in Edward medium.

The effect of inorganic salts and hydrogen ion concentration on the morphology of Mycoplasma and $L$-form colonies

Sodium chloride or $\mathrm{Li}_{2} \mathrm{SO}_{4} \cdot \mathrm{H}_{2} \mathrm{O}$ were incorporated into solid Edward medium in concentrations of $0.5,1.0,2.0$ and $3 \%(w / v)$. The plates were inoculated with Mycoplasma laidlarwii strain A and the L-form of Streptobacillus moniliformis and the growth inspected after 8 days of incubation at $37^{\circ}$. Normal growth of $M$. laidlawii strain A occurred at concentrations 0.5 and $1.0 \%(w / v)$ with both salts. At a salt concentration of $2 \cdot 0 \%(w / v)$ a partial inhibition of growth was noted; the colonies were much smaller and many lacking in peripheral zones. With $3.0 \%(w / v)$ of $\mathrm{Li}_{2} \mathrm{SO}_{4} \cdot \mathrm{H}_{2} \mathrm{O}$ none of the colonies had peripheral zones, while $\mathrm{NaCl}$ at a similar concentration inhibited growth completely. The growth of the L-form of S. moniliformis was completely inhibited with $2.0 \%$ and $\mathbf{3 . 0} \%$ concentration of both salts. At a salt concentration of $1 \%(\mathrm{w} / \mathrm{v})$ most colonies appeared with central zones only, while $0.5 \%(w / v)$ of $\mathrm{NaCl}$ or $\mathrm{Li}_{2} \mathrm{SO}_{4} \cdot \mathrm{H}_{2} \mathrm{O}$ gave normal colonies. 
The $\mathrm{pH}$ value of the medium has a marked influence on the growth of Mycoplasma organisms (Edward, 1954; Peoples, Morton \& Feo, 1957). The growth and colony morphology of Mycoplasma laidlawii strain A and the L-form of Streptobacillus moniliformis were tested at various $\mathrm{pH}$ values of Edward medium (Fig. 5). M. laidlaroii strain A did not grow at $\mathrm{pH}$ values below 6.85; optimal growth occurred at pH 7.8. Increasing the $\mathrm{pH}$ above 8.0 decreased growth, causing most colonies to appear without peripheral zones. At $\mathrm{pH}$ 8.55 peripheral zones were absent from all colonies. The L-form of $S$. moniliformis was less sensitive to $\mathrm{pH}$ changes than the Mycoplasma, but inhibition of growth also occurred at low or high pH values, accompanied by the appearance of sznall colonies without peripheral zones.

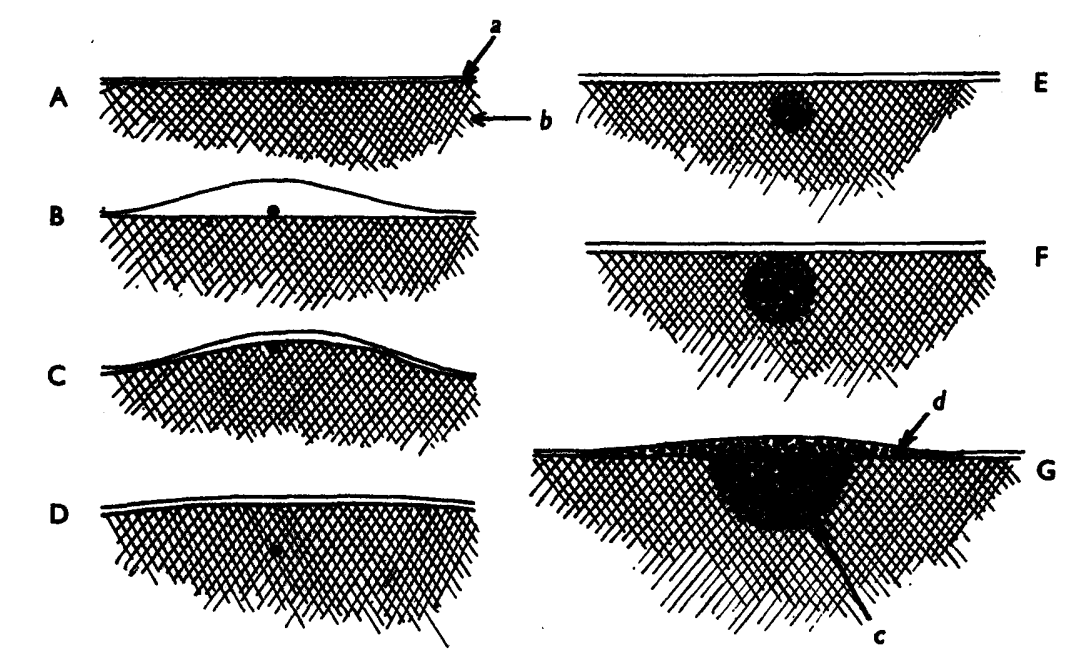

Fig. 6. Diagram to illustrate the development of Mycoplasma and L-form colonies. (A) Vertical section through the agar before inoculation : $a$, free water film; $b$, network of agar fibrils. (B) A drop containing a viable particle of Mycoplasma or $L$-form is placed on the agar. (C) C. 15 min. after inoculation : the drop is absorbed by the agar forming a slight swelling. (D) C. 8-6 hr. after inoculation: the viable particle has penetrated into agar. (E) C. $18 \mathrm{hr}$. after inoculation : a small ball-like colony has been formed inside the agar. (F) C. $24 \mathrm{hr}$. after inoculation: the ball-like colony approaches the agar surface. (G) C. 24$48 \mathrm{hr}$. after inoculation: the growth spreads into the free water film forming the peripheral zone: $c$, central zone; $d$, peripheral zone.

\section{DISCUSSION}

On the basis of our findings and those already reported by others, we are able to propose a possible mechanism for the formation of Mycoplasma and L-form colonies (shown schematically in Fig. 6). Under normal conditions the multiplication of these organisms begins inside the agar. The inoculated organisms are drawn into the agar gel together with the water surrounding them. The dried agar surface has strong imbibition properties, and water is absorbed quickly by capillary forces (Porter, 1946). This can be demonstrated by placing a drop of water on the surface of a dry agar plate; the drop is absorbed within a few minutes, resulting in a slight swelling of the agar at the drop area. When the agar surface is moist the absorption of additional water is decreased or even abolished. Therefore penetration of the organisms is very slight, or absent altogether, resulting in the formation of surface 
colonies with small or no central zones (Pl. 2, figs. 11, 13). Hence, the forces responsible for drawing the micro-organisms into the agar are the capillary forces of the agar gel. The determinant factors of the actual penetration, however, are the very small dimensions and the plastic nature of the Mycoplasma and L-forms, allowing these organisms to enter between the agar fibrils and to move in the interstices of the fibrillar network of the agar gel.

Klieneberger-Nobel (1956) showed by filtration experiments and electron microscopy that $\mathbf{L}$-form cultures contain viable particles of various sizes, the smallest of which have a diameter of about $300 \mathrm{~m} \mu$ and occur only in small numbers. In contrast, cultures of Mycoplasma are composed mainly of very small viable particles, which have an average diameter of about $100-150 \mathrm{~m} \mu$. These differences between L-forms and Mycoplasma might explain the results shown in Table 1. Most viable particles of Mycoplasma penetrated into the agar, whereas only a part of the viable particles of the $\mathbf{L}$-forms showed this ability.

The absence of motility in Mycoplasma and L-forms (Klieneberger-Nobel, 1954, 1960) excludes the possibility of active penetration of the organisms into the agar. Gravity forces do not appear to play any role in the penetration since this process is not affected by incubating the inoculated plates upside down. Actinomycetes are known to penetrate into the agar by their filamentous growth (Knaysi, 1951). Some Mycoplasma species also produce filaments and Freundt (1960) has expressed the opinion that this is a common feature of all Mycoplasma species. The possibility that the Mycoplasma penetrate into the agar through the formation of filaments is, however, disproved by the fact that penetration is accomplished within a few hours after inoculation, before the occurrence of any growth and multiplication (Table 1).

It is further shown by our results that the multiplication of the Mycoplasma and L-forms begins inside the agar at various distances from the surface, depending on the initial depth of penetration of the viable particle giving rise to the colony. During the process of multiplication the organisms do not push aside the agar, because they can penetrate and occupy the interstices between the agar fibrils. This is demonstrated by the finding that the growth of Mycoplasma and L-forms in the agar results in the formation of firm ball-like colonies instead of the lens-shaped colonies formed by the usual Eubacteria inside the agar (Pl. 3, figs. 13-15). The formation of lens-shaped deep agar colonies is explained by the inability of the usual bacteria to penetrate into the interfibrillar spaces, therefore causing the agar to split during the multiplication; the colony then spreads along this split (Knaysi, 1951). Staphylococci and streptococci do not penetrate into the agar, as shown in Table 1. It may be concluded that the central zone with Mycoplasma is a ball composed of a network of agar fibrils, with the soft micro-organisms occupying the interstices. The agar fibrils are responsible for the firmness of the central zone.

The ball-like growth inside the agar continues to grow and expand in all directions until it reaches the agar surface which is usually covered by a thin film of free water (Knaysi, 1951). At this stage the organisms spill into this free water film, forming the peripheral zone. The surface tension of the water is responsible for the circular shape of the peripheral zone. The latter is very soft because it does not contain agar, in contrast to the firm central zone. Cotton-wool fibres on the agar cause the peripheral zones of adjacent colonies to lose their round form and to spread along the fibres due to changes in the surface tension of the water surrounding the 
fibres. This is further evidence for the location of the peripheral zone in the free water film of the agar.

The dimensions of the peripheral zone are determined by the thickness of the free water film. The thinner the film, the smaller will be the peripheral zone, and the colony as a whole. This explains, at least partly, the importance of a fresh medium and a humid atmosphere for optimal growth of Mycoplasma (Edward, 1954).

It is interesting to note that the large bodies, typical of L-forms and Mycoplasma, appear only at the peripheral zone of the colony (Liebermeister, 1960). This phenomenon might be explained by remembering that inside the agar the organisms are pressed together by the agar fibrils, whereas in the peripheral zone they are free to expand either by growth of the cytoplasm, or by swelling due to low osmotic pressure of the free water film.

The necessity of a fibrillar structure of the medium for the formation of the central zone was demonstrated with the cellulose pellicle of Acetobacter xylinum, known to be composed of a network of cellulose fibrils (Mühlethaler, 1949). The typical morphology of Mycoplasma and L-form colonies on gelatin medium apparently depends also on the fibrillar nature of this gel. Hayflick \& Stinebring (1960) reported the formation of typical Mycoplasma colonies on the fibrillar plasma clot. On the other hand coagulated serum, which is not fibrillar, does not allow penetration of the organisms and the typical 'fried egg' colony does not appear with this medium (Dienes, 1960).

Apparently the wider interstices between the cellulose and gelatin fibrils, compared to agar at a concentration of $1.25 \%(w / v)$, enable the organisms to penetrate deeper into the cellulose and gelatin media. Thus, the inside growth frequently cannot reach the surface and grows into a ball-like colony inside the medium. The same may happen when the concentration of the agar in Edward medium is decreased. The wider interstices between the fibrils at low agar concentrations explain also the smallness and the irregular boundaries of the central zones observed at these concentrations. The larger viable particles of the L-forms (Klieneberger-Nobel, 1956) apparently do not penetrate quite so deeply into the agar (Table 1), thus explaining the development of large central zones and the appearance of peripheral zones at lower agar concentrations than with the Mycoplasma. The decrease of the peripheral zone dimensions observed at high agar concentrations might be explained by the diminution of the free water film under these conditions (Knaysi, 1951). Increasing the agar concentration also causes decrease of the central zone diameter, apparently due to the inhibition of penetration of the organisms into the medium.

It is noteworthy that when the medium becomes unfavourable for growth, as in the presence of high concentrations of inorganic salts or unsuitable hydrogen ion concentrations, the peripheral zone is the first to disappear, presumably because the initial ball-like growth inside the agar is not big enough to reach the surface and form the peripheral zone. This observation might explain the formation of the T-colonies of Mycoplasma (Shepard, 1960), which are composed apparently only of central zones embedded in the agar (Hayflick \& Stinebring, 1960). This is due to the unsuitability of the medium to support optimal growth of these exacting Mycoplasma strains. This was also our experience while testing defined media for Mycoplasma. Poor media only gave rise to this type of agar-embedded colonies without peripheral zones. 
Our thanks are due to Dr M. Benziman for supplying us with the cellulose pellicle of Acetobacter xylinum.

This work was supported in part by a grant from the Hebrew UniversityHadassah Medical School Research Fund.

\section{REFERENCES}

Buther, M. \& Knight, B. C. J. G. (1960a). The measurement of the growth of Mycoplasma in liquid media. J. gen. Microbiol. 22, 478.

Burtier, M. \& KNIGHT, B. C. J. G. (1960b). The survival of washed suspensions of Mycoplasma. J. gen. Microbiol. 22, 470.

Dienes, L. (1945). Morphology and nature of the pleuro pneumonia group of organisms. J. Bact. 50, 441.

Dienes, L. (1960). Controversial aspects of the morphology of PPLO. Ann. N.Y. Acad. Sci. 79, 856.

EDWARD, D. G. ff. (1954). The pleuro pneumonia group of organisms: a review, together with some new observations. J. gen. Microbiol. 10, 27.

Freund, E. A. (1960). Morphology and classification of the PPLO. Ann. N.Y. Acad. Sci. 79, 812.

HAYruick, L. \& Strnkabring, W. R. (1960). Intracellular growth of pleuro pneumonialike organisms (PPLO) in tissue culture and in ovo. Ann. N.Y. Acad. Sci. 79, 433.

Hestrin, S. \& Schramm, M. (1954). Synthesis of cellulose by Acetobacter xylinum. 2. Preparation of freeze-dried cells capable of polymerizing glucose to cellulose. Biochem. J. 58, 845.

KunENEBerger, E. (1984). The colonial development of the organisms of pleuropneumonia and agalactia on serum agar and variations of the morphology under different conditions of growth. J. Path. Bact. 39, 409.

KLIENEBerger-Nober, E. (1954). Micro-organisms of the pleuropneumonia group. Biol. Rev. 29, 154.

KuIENeberger-Nober, E. (1956). Uber die Wesensverschiedenheit der peripneumonieähnlichen Organismen und der L-phase der Bakterien. Zbl. Bakt. Abt. I. Orig. 165, 329.

KuIENEBerger-Nobel, E. (1960). L-forms of bacteria in The Bacteria, $A$ Treatise on Structure and Function. Ed. I. C. Gunsalus and R. Y. Stanier, 1, 361. New York: Academic Press Inc.

KNays1, G. (1951). Elements of Bacterial Cytology, 2nd ed. Ithaca: Comstock Publishing Co. Inc.

Liebermeister, K. (1960). Morphology of the PPLO and L-forms of Proteus. Ann. N.Y. Acad. Sci. 79, 326.

Mühlethaler, K. (1949). The structure of bacterial cellulose. Biochim. Biophys. Acta, 3, 527.

Peoples, D. M., Morton, H. E. \& Feo, L. G. (1957). Unusual pleuropneumonia-like organisms isolated in a study of Trichomonas vaginalis from cases of chronic urethritis. J. Bact. 73, 898.

Ponter, J. R. (1946). Bacterial Chemistry and Physiology, p. 28. New York: John Wiley and Sons, Inc.

ShePARD, M. C. (1960). Recovery, propagation and characteristics of T-strain PPLO isolated from human cases of nongonococcal urethritis. Ann. N.Y. Acad. Sci. 79, 397.

EXPLANATION OF PLATES

Prate 1

Figs. 1-8. The development of Mycoplasma laidlareii strain A colonies on Edward medium after 24,48 and $96 \mathrm{hr}$. incubation at $37^{\circ} . \times 50$.

Figs. 4-6. The development of the L-form of Streptobacillus moniliformis colonies. Medium and incubation periods as above. $\times 50$. 

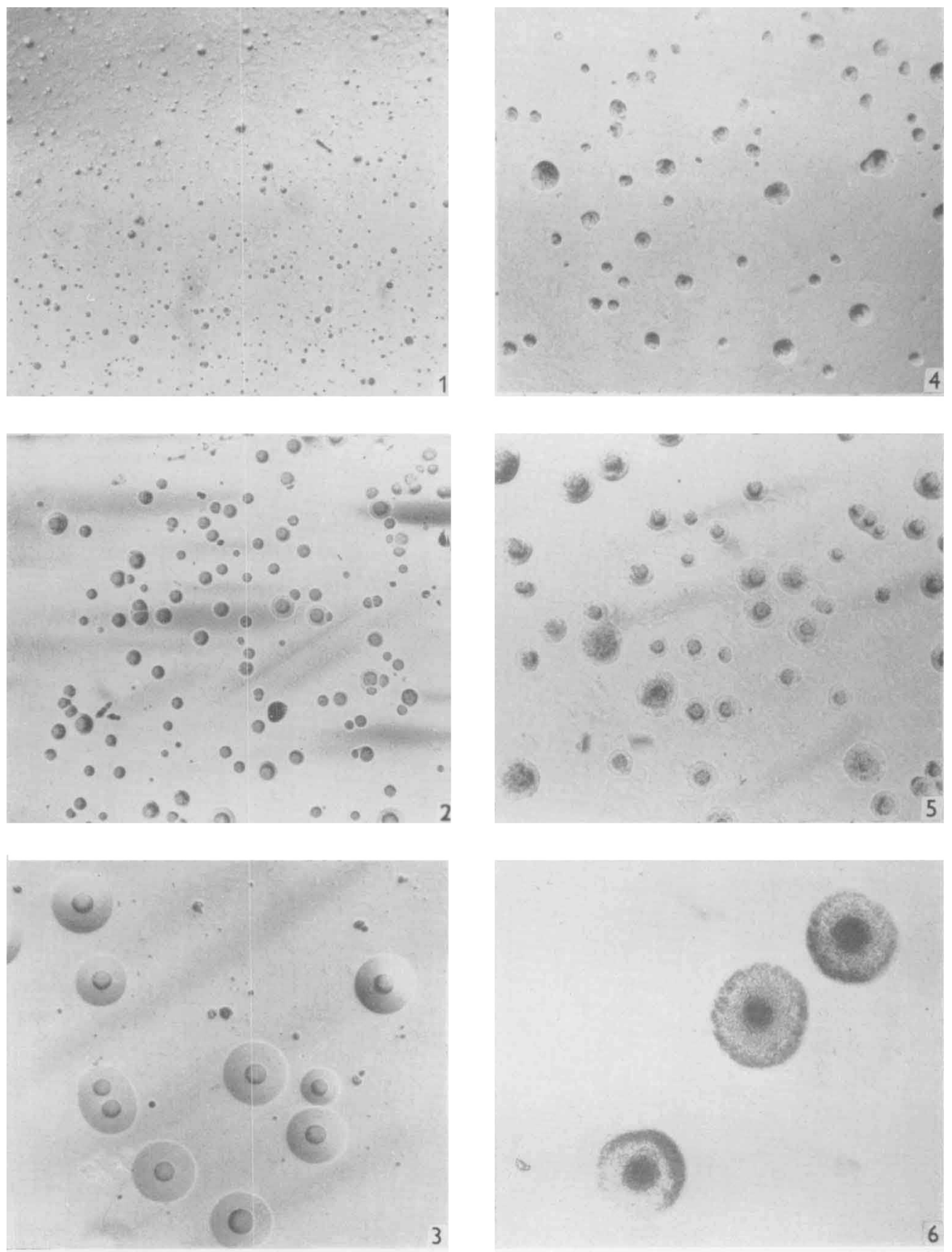

S. RAZIN AND O. OLIVER 
Journal of General Microbiology, Vol. 24, No. 2

Plate 2
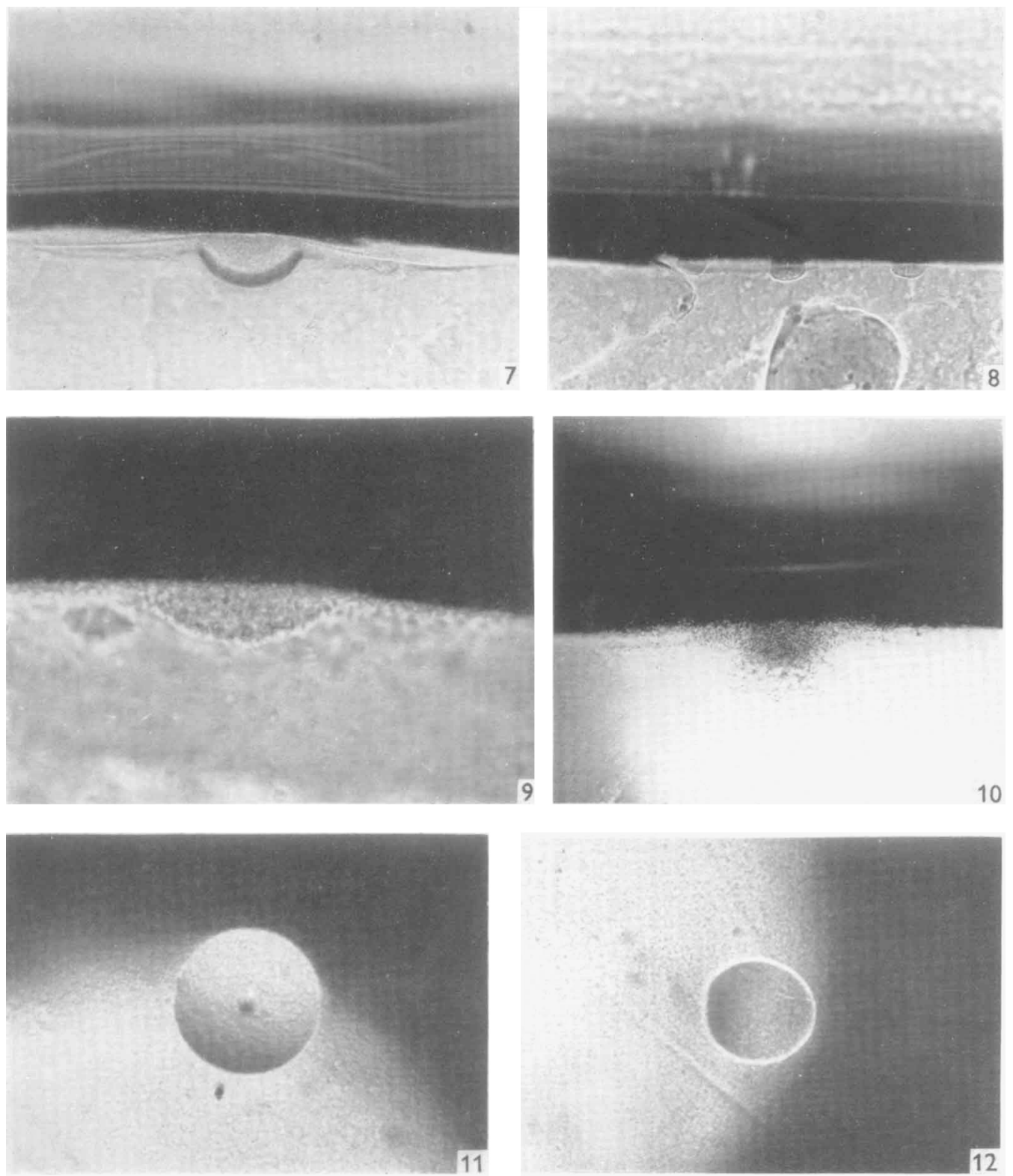

S. RAZIN AND O. OLIVER 

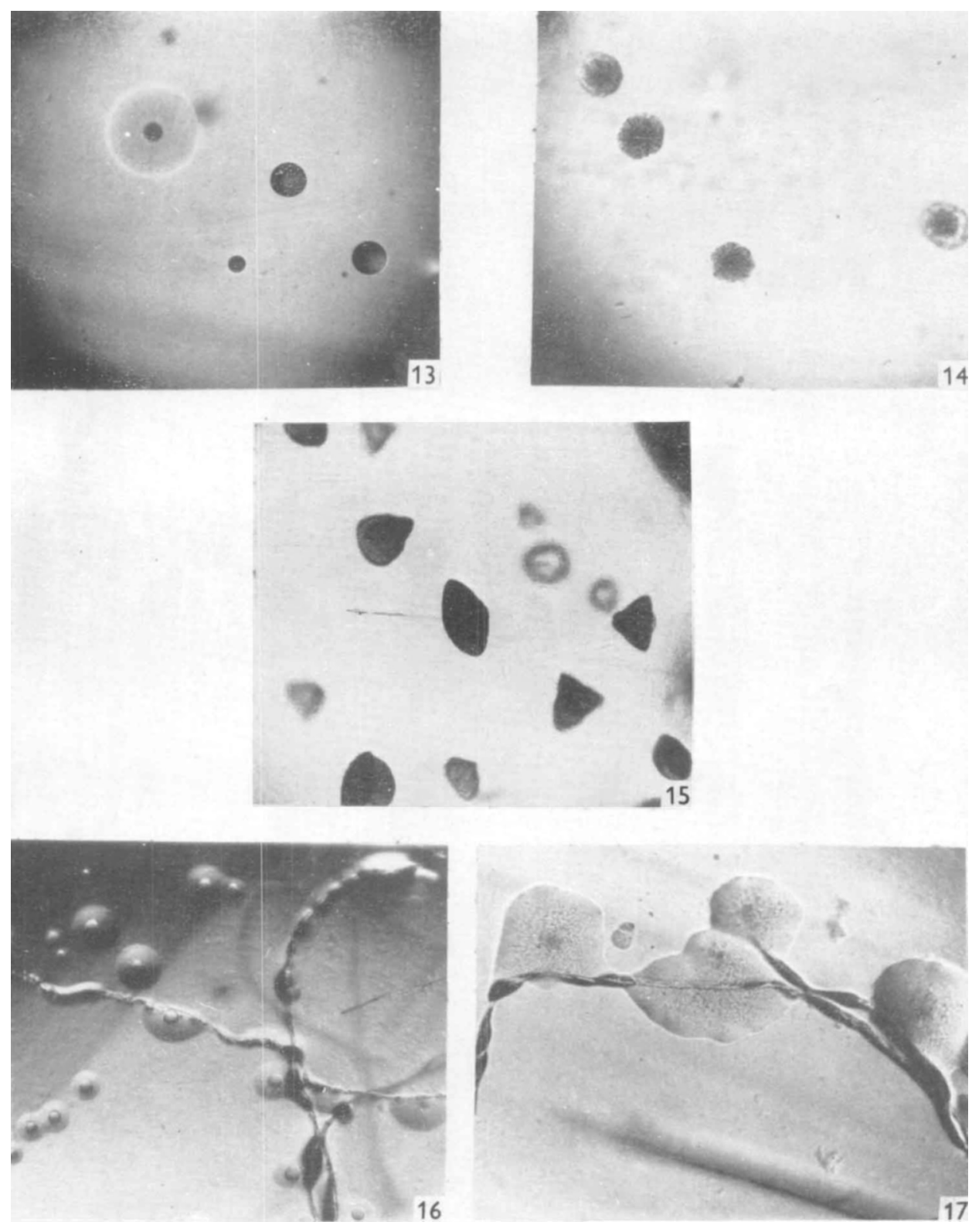

S. RAZIN AND O. OLIVER 


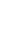


Figs. 7-10. Vertical sections of colonies.

\section{Prate 2}

Fig. 7. Mycoplasma laidlawii strain A. $\times 150$.

Fig. 8. Mycoplasma mycoides var. capri. $\times 150$

Fig. 9. Mycoplasma gallisepticum. $\times 600$.

Fig. 10. L-form of Proteus. $\times 150$.

Figs. 11, 12. Colonies of Mycoplasma laidlawii strain A grown on moist Edward agar. $\times 50$.

\section{Plate 8}

Fig. 18. Deep Edward agar colonies of Mycoplasma laidlawii strain A. Including one surface colony with a peripheral zone. $\times 50$.

Fig. 14. Deep Edward agar colonies of the L-form of Streptobacillus moniliformis. One small surface colony is seen at the right end of the photograph. $\times 50$.

Fig. 15. Deep Edward agar colonies of Staphylococcus aureus. $\times 50$.

Fig. 16. Mycoplasma laidlazwii strain A colonies touching cotton-wool fibres on the surface of Edward medium. $\times 50$.

Fig. 17. L-form of Streptobacillus moniliformis colonies touching cotton-wool fibres on the surface of Edward medium. $\times 50$. 